\title{
q DEFORMATION BY INTERTWINNING WITH APPLICATION TO THE SINGULAR OSCILLATOR
}

\author{
HARET C. ROSU ${ }^{\dagger}$ 的 CARLOS CASTRO* \\ $\dagger$ Instituto de Física, Universidad de Guanajuato, Apdo Postal E-143, \\ León, Guanajuato 37150 Mexico \\ * Center for Theoretical Studies of Physical Systems, Clark Atlanta Uni- \\ versity, Atlanta, Georgia 30314 USA
}

\begin{abstract}
We present a version of $q$-deformed calculus based on deformed counterparts of Darboux intertwining operators. The case in which the deformed transformation function is of the vacuum type is detailed, but the extension to counterparts of excited states used as Darboux transformation functions is also formally discussed. The method leads to second-order Fokker-Planck-like deformed operators which may be considered as supersymmetric partners, though for a sort of $q$-deformed open systems, i.e., those possessing $q$ nonlocal drift terms, potential part, as well as $q$-spreaded vacuum fluctuations. The undeformed limit corresponds to the conservative case, since all $q$ nonlocalities wash out. The procedure is applied to the $x^{-2}$ singular oscillator, for which we also present a formal $q$ generalization of the Bagrov-Samsonov coherent states.
\end{abstract}

Accepted at Phys. Lett. A on Nov. 29, 1999 quant-ph/9808021 v4].

\section{INTRODUCTION}

At the present time, the $q$-deformed calculus is widely applied to many physical problems 1 , but although there are many advances in mathematics, the attention of physicists is still mainly captured by the $q$ harmonic oscillators introduced by Arik and Coon, Biedenharn, and Macfarlane 2. In this work, we present a general $q$-deformed framework that next we apply to the singular oscillator, which is based on the idea of using, as fundamental tools, some deformed counterparts of the intertwining operators encountered in the area of Darboux transformations. We have been motivated to write this paper by recent results of Bagrov and Samsonov 3 , who provided an important extension of the Darboux method by showing that the application of the Darboux transformation/intertwining operator to the coherent states of the initial quantum system gives a set of states which may be treated as coherent states of the transformed system. Moreover, employing the example of the singular oscillator, Samsonov $\mathrm{Q}$ has shown that the Darboux transformation for this case translates into the transformation of the Kähler potential corresponding to a

\footnotetext{
${ }^{a}$ Electronic mail address: rosu@ifug3.ugto.mx

${ }^{b}$ Electronic mail address: castro@hubble.cau.edu
} 
manifold of varying curvature. This leads to a calculable distortion of the initial phase space.

Our basic idea is to use the factorization property of deformed analogues of the intertwining operators to build second-order deformed operators which are of the Fokker-Planck type, containing $q$ drift ( $q$ first derivative) sectors, as well as contributions that, when going to particular quantum mechanical problems, may be shown to be modifications of the potential and zero point sectors. In particular, we obtain a sort of $q$ nonlocal "spatially-spreaded" zero point sector. In the undeformed limit $q \rightarrow 1$, the $q$ drift sectors disappear, while the other sectors get the conventional forms of the original Hamiltonian system as we show for the singular oscillator.

The organization of the paper is the following. In the next section we present our $q$-deformed intertwining method in general terms. Then, in section 3 , the theory of singular oscillator is briefly reviewed, together with the recently introduced Darboux coherent states. In section 4 , the $q$-deformed intertwining method is applied to the singular oscillator, introducing $q$-deformed Darboux coherent states as well, and we end up with the conclusion.

\section{2. q DEFORMATION BY INTERTWINING}

Here we present the general framework of the $q$-deformed calculus based on deformed counterparts of the intertwining operators, which in standard supersymmetric quantum mechanics are also known as supersymmetric charges or supercharges. When the method is applied to particular cases, such as the singular oscillator (see section 4), one can get more insight on the features of the procedure.

The independent variable is still the real commutative half line, and therefore, from this point of view, our version of the deformed calculus is similar to that previously used by some authors who deformed the Coulomb problem $\mathrm{E}$. We shall employ symmetric definitions of the $q$ number $[x]_{q}=\frac{q^{x}-q^{-x}}{q-q^{-1}}$ and $q$ derivative

$$
D_{q} f(x)=\frac{f(q x)-f\left(q^{-1} x\right)}{x\left(q-q^{-1}\right)} .
$$

Some of the basic rules of Jackson's calculus 1 , such as $D_{q} x^{n}=[n]_{q} x^{n-1}, D_{q}^{2} x^{n}=$ $[n]_{q}[n-1]_{q} x^{n-2}, D_{q}(F G)=\left(D_{q} F\right) G(q x)+F\left(q^{-1} x\right)\left(D_{q} G\right)$ have been used in the calculations. The definition of the $q$ exponential is

$$
e_{q}(x)=\sum_{n=0}^{\infty} \frac{x^{n}}{[n]_{q} !},
$$

which reduces to the usual exponential function as $q \rightarrow 1$, and is invariant under $q \rightarrow q^{-1}$.

As well known, in supersymmetric quantum mechanics a ground state (or vac-

uum) can be written as $u_{0}(x) \propto e^{\int^{x} W}$, where $W$ is a solution of the corresponding Riccati equation usually called superpotential. The main idea for the following is 
to consider a deformed ground state $u_{0 q}$, in the sense of deforming the exponential as in (2), and exploit the factorization property of first-order deformed operators (supercharges) of the form

$$
T_{01}^{q}=D_{q}-\frac{D_{q} u_{0 q}}{u_{0 q}}
$$

and

$$
T_{02}^{q}=-D_{q}-\frac{D_{q} u_{0 q}}{u_{0 q}}
$$

These operators have been written by analogy to the continuous intertwining operators. Thus, by employing the factorization procedure, we would like to see what sort of second-order deformed operators we shall obtain from the products $T_{02}^{q} T_{01}^{q}$ and $T_{01}^{q} T_{02}^{q}$, respectively.

After a straightforward calculation we get the following operators

$£_{0 i}^{q} \equiv T_{02}^{q} T_{01}^{q}=-D_{q}^{2}-\left[\left(\frac{D_{q} u_{0 q}}{u_{0 q}}\right)_{(x)}-\left(\frac{D_{q} u_{0 q}}{u_{0 q}}\right)_{(x / q)}\right] D_{q}+\left[\left(\frac{D_{q} u_{0 q}}{u_{0 q}}\right)_{\rightarrow x}^{2}+D_{q}\left(\frac{D_{q} u_{0 q}}{u_{0 q}}\right)_{\rightarrow q x}^{2}\right]$

and

$£_{0 f}^{q} \equiv T_{01}^{q} T_{02}^{q}=-D_{q}^{2}+\left[\left(\frac{D_{q} u_{0 q}}{u_{0 q}}\right)_{(x)}-\left(\frac{D_{q} u_{0 q}}{u_{0 q}}\right)_{(x / q)}\right] D_{q}+\left[\left(\frac{D_{q} u_{0 q}}{u_{0 q}}\right)_{\rightarrow x}^{2}-D_{q}\left(\frac{D_{q} u_{0 q}}{u_{0 q}}\right)_{\rightarrow q x}^{2}\right]$.

If one multiplies (5) and (6) by (-1) a quite natural interpretation of the operators $-£_{0 i}^{q}$ and $-£_{0 f}^{q}$ is as $q$-deformed Fokker-Planck operators. They may be considered as supersymmetric partners describing a pair of open systems that are connected in an algebraic manner. Along with the $q$ kinetic $D_{q}^{2}$ term, they contain a $D_{q}$ drift part, and a nonoperatorial potential contribution. The latter two sectors have been delimited by square brackets in (5) and (6). The directional subindices indicate the argument of the solution to which the nonoperatorial terms are to be attached, whereas the subindices in the drift coefficients show explicitly their arguments. The main feature of the two operators is the $q$ nonlocality. Using the Fokker-Planck interpretation, one may say that the coefficient of the first derivative term is the $q$ derivative of the drift potential.

The procedure can be extended easily to models for which the deformed Darboux transformation functions correspond to excited states of the form

$$
\psi_{n q} \propto P_{n}\left(x^{2} ; q\right) e_{q}\left(-\beta x^{2}\right)
$$

where $P_{n}\left(x^{2} ; q\right)$ is a deformed counterpart of the special polynomial $P_{n}\left(x^{2}\right)$. As a matter of fact, a third monomial factor is also allowed in (7). In order to avoid any singularities in the undeformed limit, it is convenient to perform an $i$-rotation $x \rightarrow i x$, leading to

$$
u_{p q}(x) \propto P_{p q}(y) \exp _{q}\left(\beta x^{2}\right), \quad y=-x^{2} / 2, \quad p=0,1,2, \ldots
$$


and use $u_{p q}(x)$ as the Darboux transformation function since $P_{p q}(y) \neq 0 \forall x \neq 0$ when $y=-x^{2} / 2(<0)$ and, hence, the function $u_{p q}(x)$ is nodeless for $x>0$. The intertwining operators can be calculated according to $T_{p 1}^{q}=D_{q}-\frac{D_{q} u_{p q}}{u_{p q}}$ and $T_{p 2}^{q}=$ $-D_{q}-\frac{D_{q} u_{p q}}{u_{p q}}$, and again by exploiting the factorization property one is led to secondorder deformed operators of a more complex structure than (5) and (6). Formally, one should merely replace the subindex 0 with $p$ in (5) and (6). We shall apply this extension to the singular oscillator that belongs to the class of models possessing excited states for which the $i$ rotation works.

\section{THE SINGULAR OSCILLATOR}

The $x^{-2}$ singular quantum oscillator appears in many physical problems, such as diatomic and polyatomic molecules 6 , the Calogero problem 6 , fractional statistics and anyons 8 , two-dimensional QCD 9, and others. Very recently, Dodonov, Man'ko and Rosa 10, following Combescure's suggestion 11, presented a detailed study of the more general time-dependent quantum singular oscillator as a model of a two-ion trap. Especially in the latter case, various coherent states are an important topic, because their mesoscopic superpositions commonly known as Schrödinger cat-like states can be studied experimentally, although those of Darboux type 12 have not yet been investigated in the physics of traps. Moreover, in the same context, discrete irregular effects of both (quasi)periodic $\delta$-kick type and other discrete spatial type may prove important since, as remarked in 10 , the singular oscillator system lies at the border between linearity and nonlinearity, and chaotic behavior is a characteristic feature of the overwhelming majority of deterministic nonlinear dynamical systems 13.

We now briefly sketch the approach of Bagrov and Samsonov for a Darboux class of coherent states 3. The Hamiltonian of the (half-line/radial) quantum oscillator with $x^{-2}$ singular part

$$
h_{0}=-d^{2} / d x^{2}+x^{2} / 4+b / x^{2}, \quad b>0, \quad x \in[0, \infty)
$$

has the $s u(1,1)$ dynamical symmetry algebra and therefore is exactly solvable. The more general time-dependent singular oscillator has been already solved in 1971 14. In coordinate representation the generators of this algebra are expressed in terms of the harmonic oscillator annihilation $a$ and creation $a^{+}$operators

$$
\begin{gathered}
K_{0}=\frac{1}{2} h_{0}, \quad K_{+}=\frac{1}{2}\left[\left(a^{+}\right)^{2}-b / x^{2}\right], \quad K_{-}=\frac{1}{2}\left[a^{2}-b / x^{2}\right], \\
a=d / d x+x / 2, \quad a^{+}=-d / d x+x / 2,
\end{gathered}
$$

and satisfy the standard commutation relations

$$
\left[K_{0}, K_{ \pm}\right]= \pm K_{ \pm}, \quad\left[K_{-}, K_{+}\right]=2 K_{0}
$$

For an irreducible representation the corresponding $s u(1,1)$ Casimir operator takes the constant value $\mathcal{C}=\frac{1}{2}\left[K_{+} K_{-}+K_{-} K_{+}\right]-K_{0}^{2}=3 / 16-b / 4=k(1-k)$. The value 
of $k=1 / 2+(1 / 4) \sqrt{1+4 b}$ defines the ground state (vacuum) energy $E_{0}=2 k$. The vacuum state $|0\rangle$ is defined by the equations: $K_{-}|0\rangle=0$ and $K_{0}|0\rangle=k|0\rangle$. A very interesting feature of the ground state of the singular oscillator is the presence of a centrifugal barrier of the type $b_{0} / x^{2}$, where $b_{0}=(2 k-3 / 2)(2 k-1 / 2)$ that may be considered as produced by the zero point fluctuations.

Other discrete Fock eigenfunctions $|n\rangle$ of the Hamiltonian $h_{0}$ are defined by applying successive powers of the raising operator $K_{+}$, and in the coordinate representation they have the form

$$
\psi_{n}(x)=\langle x \mid n\rangle=\left[n ! 2^{1-2 k} \Gamma^{-1}(n+2 k)\right]^{1 / 2} x^{2 k-1 / 2} L_{n}^{2 k-1}\left(x^{2} / 2\right) \exp \left(-x^{2} / 4\right),
$$

where $L_{n}^{2 k-1}\left(x^{2} / 2\right)$ are the associated Laguerre polynomials.

Passing now to the $s u(1,1)$ coherent states, we first mention their rich variety in the literature 15, although one may consider as the most appropriate those introduced by Barut and Girardello 16 as the eigenstates of the Weyl lowering operator $K_{-}$of the $s u(1,1)$ algebra. In the approach of Bagrov and Samsonov the coherent states are the kets $|z\rangle=N_{0 z} \sum_{n=0}^{\infty} a_{n} z^{n}|n\rangle$, where

$$
N_{0 z}^{2}=(1-z \bar{z})^{2 k}, \quad a_{n}=(-1)^{n} \sqrt{\frac{\Gamma(2 k+n)}{n ! \Gamma(2 k)}}, \quad|z|<1 .
$$

The linear manifold spanned by the coherent vectors $\{|z\rangle\}$ forms an everywhere dense and overcomplete set in the Hilbert space $\mathcal{H}_{1}$ with the identity decomposition $\int_{|z|<1}|z\rangle\langle z| d \mu(z)=1$, of measure $d \mu(z)=\frac{2 k-1}{\pi}(1-z \bar{z})^{-2} d z d \bar{z}$.

The Fourier coefficients $c_{n}$ of a vector $|\psi\rangle \in \mathcal{H}_{1}$ with respect to the basis $\{|n\rangle\}$ define a holomorphic representation $\psi(z)$ of the vector $|\psi\rangle$ in the space of functions which are holomorphic in the unit disk

$$
\langle\bar{z} \mid \psi\rangle=N_{0 z} \psi(z), \quad \psi(z)=\sum_{n=0}^{\infty} a_{n} c_{n} z^{n} .
$$

Let $\mathcal{L}$ be the linear span of entire analytic functions $\psi(z)$ defined in the unit disk such that $\int_{|z|<1}|\psi(z)|^{2}\left(1-|z|^{2}\right)^{2 k} d \mu(z)<\infty$. If in $\mathcal{L}$ one defines the inner product

$$
\left\langle\psi_{1}(z) \mid \psi_{2}(z)\right\rangle \equiv \int_{|z|<1} e^{-f^{(0)}} \bar{\psi}_{1}(z) \psi_{2}(z) d \mu(z)=\left\langle\psi_{1} \mid \psi_{2}\right\rangle
$$

where $\left\langle\psi_{1} \mid \psi_{2}\right\rangle$ is the inner product in the Hilbert space $\mathcal{H}_{1}$ then the completion of $\mathcal{L}$ with respect to this inner product gives the Hilbert space $\mathcal{H}_{2}$. The function $f^{(0)}=\ln |\langle 0 \mid z\rangle|^{-2}=\ln (1-z \bar{z})^{-2 k}$ is the Kähler potential in the unit disk. The corresponding metric has the form

$$
d s^{2}=g_{z \bar{z}} d z d \bar{z}, \quad g_{z \bar{z}}=\frac{\partial^{2} f^{(0)}}{\partial z \partial \bar{z}}=\frac{2 k}{(1-z \bar{z})^{2}} .
$$


The imaginary part of this metric defines a symplectic 2-form $\omega=-i g d z \wedge d \bar{z}$ and consequently a Poisson bracket $\left\{F_{1}, F_{2}\right\}$ of the functions $F_{1}$ and $F_{2}$ defined on the unit disk. The unit disk has now all the requirements of a phase space of constant Gauss curvature $\mathcal{K}^{(0)}=-\frac{2}{k}$.

In order to get Darboux-transformed coherent states one should use the standard Darboux transformation operator $T=-t_{u}(x)+d / d x=-u^{\prime}(x) / u(x)+d / d x$, where the prime denotes the derivative with respect to $x$. When acting on the solutions $\psi_{n}(x)$ of the initial Schrödinger equation $h_{0} \psi_{n}(x)=E_{n} \psi_{n}(x), \quad E_{n}=2 n+2 k$, it transforms them into the solutions of another Schrödinger equation $h_{1} \varphi_{n}(x)=$ $E_{n} \varphi_{n}(x), \varphi_{n}(x)=N_{n} T \psi_{n}(x)$, with the same eigenvalues $E_{n}$. The factor $N_{n}$ is introduced to ensure that the states $\varphi_{n}$ are normalized to unity. The new exactly solvable Hamiltonian has the form $h_{1}=h_{0}+A(x)$, where the potential difference is of Darboux type $A(x)=-2(\ln u)^{\prime \prime}$. The function $u=u(x)$ is the Darboux transformation function, being a solution of the initial Schrödinger equation $h_{0} u(x)=\alpha u(x)$ with $\alpha \leq E_{0}$. Samsonov treated the simpler case when $\alpha<E_{0}$, (more exactly he used $\alpha=-2(k+p)$ and an analytic continuation in order to work with a nodeless transformation function). Thus, $u(x) \neq 0 \forall x \in(0, \infty)$ and $1 / u(x)$ is not a square integrable function in the interval $[0, \infty)$. In this case $u \notin \mathcal{H}_{1}$ and the set $\left\{\left|\varphi_{n}\right\rangle\right\}$ constitutes a complete basis in the Hilbert space $\mathcal{H}_{1}$ provided the initial system $\left\{\left|\psi_{n}\right\rangle\right\}$ is complete. In terms of the supersymmetric quantum mechanics this case corresponds to a broken supersymmetry. The operator $T^{+}=-t_{u}(x)-d / d x$ performs the transformation in the inverse direction $\left|\psi_{n}\right\rangle=N_{n} T^{+}\left|\varphi_{n}\right\rangle$, and together with $T$ participates in the factorizations $T^{+} T=h_{0}-\alpha, \quad T T^{+}=h_{1}-\alpha$. The $T$ and $T^{+}$operators are well defined $\forall \psi \in \mathcal{H}_{1}$ and are conjugated to each other with respect to the inner product in the $\mathcal{H}_{1}$ space.

We can now define, following Bagrov and Samsonov 目, the Darboux-transformed

coherent states $\varphi_{z}(x)=N_{1 z} T \psi_{z}(x)=N \sum_{n=0}^{\infty} b_{n} z^{n} \varphi_{n}$, where $N=N_{0 z} N_{1 z} / N_{0}, \quad b_{n}=$ $a_{n} N_{0} / N_{n}$. From the factorization properties of the $T$ operators one can derive the value of the normalization constant $N_{1 z}^{2}=(1-z \bar{z}) /(4 k+2 p-2 p z \bar{z})$, which, as shown by Samsonov 4 , is responsible for the Gauss varying curvature of the phase space disk.

\section{4. q-DEFORMED INTERTWINING FOR THE SINGULAR OSCILLATOR}

For the singular oscillator the deformed vacuum states are of the type $u_{0 q} \propto$ $x^{\gamma} e_{q}\left(\beta x^{2}\right)$, where $\gamma=2 k-1 / 2$ and $\beta= \pm 1 / 4$ for the irregular and regular vacuum respectively. The irregular vacuum is the one not well-behaved asymptotically, but, as known, can be used as Darboux transformation function. The first-order deformed 
operators read

$$
\begin{gathered}
T_{01}^{q}=D_{q}-\frac{D_{q} u_{0 q}}{u_{0 q}}=D_{q}-\frac{[\gamma]_{q}}{x} \epsilon_{q}\left(x^{2}\right)-\beta_{q}\left(x^{2}\right) x, \\
T_{02}^{q}=-D_{q}-\frac{D_{q} u_{0 q}}{u_{0 q}}=-D_{q}-\frac{[\gamma]_{q}}{x} \epsilon_{q}\left(x^{2}\right)-\beta_{q}\left(x^{2}\right) x,
\end{gathered}
$$

where

$$
\begin{gathered}
\epsilon_{q}\left(x^{2}\right)=\frac{e_{q}\left(\beta q^{2} x^{2}\right)}{e_{q}\left(\beta x^{2}\right)} \\
\beta_{q}\left(x^{2}\right)=4 \beta q^{-\gamma}\left(\frac{q e_{q}\left(q \beta x^{2}\right)+q^{-1} e_{q}\left(q^{-1} \beta x^{2}\right)}{e_{q}\left(\beta x^{2}\right)}\right) .
\end{gathered}
$$

The second-order $q$-deformed operators are given by

$$
\begin{gathered}
£_{0 i}^{q} \equiv T_{02}^{q} T_{01}^{q}=-D_{q}^{2}-\left[\left(\frac{[\gamma]_{q}}{x}\left(\Delta \epsilon_{q}\right)+\left(\Delta \beta_{q}\right) x\right) D_{q}\right]+ \\
{\left[\left(\frac{[\gamma]_{q}^{2}}{x^{2}} \epsilon_{q}^{2}\left(x^{2}\right)+\beta_{q}^{2}\left(x^{2}\right) x^{2}\right)_{\rightarrow x}-\left(\frac{[\gamma]_{q}}{x^{2}} \epsilon_{q}\left(q^{2} x^{2}\right)-\frac{q[\gamma]_{q}}{x}\left(D_{q} \epsilon_{q}\left(x^{2}\right)\right)-q\left(D_{q} \beta_{q}\left(x^{2}\right)\right) x\right)_{\rightarrow q x}\right]} \\
+\left[\left(2[\gamma]_{q} \beta_{q}\left(x^{2}\right) \epsilon_{q}\left(x^{2}\right)\right)_{\rightarrow x}+\left(\beta_{q}\left(q^{-2} x^{2}\right)\right)_{\rightarrow q x}\right]
\end{gathered}
$$

and

$$
\begin{gathered}
£_{0 f}^{q} \equiv T_{01}^{q} T_{02}^{q}=-D_{q}^{2}+\left[\left(\frac{[\gamma]_{q}}{x}\left(\Delta \epsilon_{q}\right)+\left(\Delta \beta_{q}\right) x\right) D_{q}\right]+ \\
{\left[\left(\frac{[\gamma]_{q}^{2}}{x^{2}} \epsilon_{q}^{2}\left(x^{2}\right)+\beta_{q}^{2}\left(x^{2}\right) x^{2}\right)_{\rightarrow x}+\left(\frac{[\gamma]_{q}}{x^{2}} \epsilon_{q}\left(q^{2} x^{2}\right)-\frac{q[\gamma]_{q}}{x}\left(D_{q} \epsilon_{q}\left(x^{2}\right)\right)-q\left(D_{q} \beta_{q}\left(x^{2}\right)\right) x\right)_{\rightarrow q x}\right]} \\
+\left[\left(2[\gamma]_{q} \beta_{q}\left(x^{2}\right) \epsilon_{q}\left(x^{2}\right)\right)_{\rightarrow x}-\left(\beta_{q}\left(q^{-2} x^{2}\right)\right)_{\rightarrow q x}\right]
\end{gathered}
$$

where

$$
\Delta \epsilon_{q}=\epsilon_{q}\left(x^{2}\right)-q \epsilon_{q}\left(q^{-2} x^{2}\right)
$$

and

$$
\Delta \beta_{q}=\beta_{q}\left(x^{2}\right)-q^{-1} \beta_{q}\left(q^{-2} x^{2}\right)
$$

In (21) and (22) one can see a nonlocal potential part in the second square bracket and a nonlocal zero point contribution in the third square bracket. Moreover, in the $q \rightarrow 1$ limit, the potential and zero point sectors take forms identical to those of the undeformed singular oscillator. More precisely, the undeformed limits read $(d=d / d x)$

$$
£_{0 i}^{1} \equiv h_{0}=-d^{2}+\frac{\gamma(\gamma-1)}{x^{2}}+\beta^{2} x^{2}+\beta(2 \gamma+1)
$$


and

$$
£_{0 f}^{1} \equiv h_{1}=-d^{2}+\frac{\gamma(\gamma+1)}{x^{2}}+\beta^{2} x^{2}+\beta(2 \gamma-1) .
$$

Thus, when $q \rightarrow 1$ one gets the conservative limit of the open (driven) systems described by the operators $£^{q}$. Notice also that the difference between the Hamiltonian partners (25) and (26) can be assigned to a change of the balance between the centrifugal barrier and the vacuum fluctuations.

In the more general case of the excited states of the singular oscillator, the deformed counterparts can be written in the form

$$
\psi_{n q} \propto x^{\gamma} L_{n}^{\gamma-1 / 2}\left(x^{2} ; q\right) e_{q}\left(-\frac{1}{4} x^{2}\right)
$$

where $L_{n}^{\gamma-1 / 2}\left(x^{2} ; q\right)$ is a deformed Laguerre polynomial (see 17). One can see that we are in the class of models mentioned at the end of section 2. Thus, in order to avoid any singularities, it is convenient to perform an $i$-rotation $x \rightarrow i x$, leading to

$$
u_{p q}(x) \propto x^{\gamma} L_{p q}^{\gamma-1 / 2}(y) \exp _{q}\left(x^{2} / 4\right), \quad y=-x^{2} / 2, \quad p=0,1,2, \ldots
$$

and use $u_{p q}(x)$ as the Darboux transformation function since $L_{p q}^{\gamma-1 / 2}(y) \neq 0 \forall x \neq 0$ when $y=-x^{2} / 2(<0)$ and, hence, the function $u_{p q}(x)$ is nodeless for $x>0$.

The intertwining operators can be calculated according to $T_{p 1}^{q}=D_{q}-\frac{D_{q} u_{p q}}{u_{p q}}$ and $T_{p 2}^{q}=-D_{q}-\frac{D_{q} u_{p q}}{u_{p q}}$, and by employing the factorization property one is led to second-order deformed operators of a more complex structure than in the vacuum case.

Moreover, a whole manifold of Bagrov-Samsonov Darboux-transformed wavefunctions corresponding to the deformed number states of the singular oscillator can be introduced by applying various $T_{p 1}^{q}$ operators

$$
\phi_{n p}^{q}(x) \propto T_{p 1}^{q} \psi_{n q}(x) .
$$

The corresponding q-deformed Darboux coherent states are defined by the expansion

$$
\phi_{z p}^{q}(x) \propto \sum\left(b_{n p}^{q} z^{n}\right) \phi_{n p}^{q}(x),
$$

and they fulfill the identity decomposition of the type

$$
\int_{|z|<1}\left|\phi_{z p}^{q}\right\rangle\left\langle\phi_{z p}^{q}\right| d_{q} \nu(z)=1
$$

where the integral is of Jackson type, i.e., $\int_{0}^{a} f(x) d_{q} x=a\left(q^{-1}-q\right) \sum_{n=0}^{\infty} q^{2 n+1} f\left(q^{2 n+1} a\right)$.

The calculation of the Darboux distortion of the dissipative phase space of commutative $q$-deformed systems is beyond the scope of this work. Nevertheless, we point here that while in the conservative case, because of the isospectrality property, the Darboux distortion is consistent with the change of the Hamilton function 
in such a manner that the curves of a constant energy (i.e., the classical trajectories) in the Darboux-transformed phase space remain unchanged $\mathbb{E}$, the deformed case corresponds to driven and dissipative systems and will miss this property.

\section{CONCLUSION}

In conclusion, we presented here a new approach to a commutative $q$-deformed calculus, which is based on a special type of $q$-deformed intertwining operators. As an example, we applied our procedure to the important solvable case of the $x^{2}+x^{-2}$ singular oscillator. Mesoscopic physics is the realm of open systems, being dominated by nonlocal and decoherence effects. We have shown by Darboux methods that the $q$ deformation is a different means to introduce in the dynamics drifts and dissipation and possibly spatial discreteness (e.g., by discretizing the deformation parameter 18), all endowed with $q$ nonlocal features.

This work has been partially supported by CONACYT project 458100-5-25844E. 


\section{References}

1 L.C. Biedenharn and M.A. Lohe, Quantum Group Symmetry and q-Tensor Algebras (World Scientific, Singapore 1995).

2 M. Arik and D.D. Coon, J. Math. Phys. 17 (1976) 524; L.C. Biedenharn, J. Phys. A 22 (1989) L873; A.J. Macfarlane, J. Phys. A 22 (1989) 4581.

3 V.G. Bagrov and B.F. Samsonov, 1996 Zh. Eksp. Teor. Fiz. 109 (1996) 1105; J. Phys. A 29 (1996) 1011.

4 B.F. Samsonov, J. Math. Phys. 39 (1998) 967.

5 See for example, F.L. Chan and R.J. Finkelstein, J. Math. Phys. 35 (1994) 3273; J. Feigenbaum and P.G.O. Freund, J. Math. Phys. 37 (1996) 1602.

6 A.V. Gorokhov, Izv. Vuz. Fiz. 2 (1971) 148; A. Hartmann, Theor. Chim. Acta 24 (1972) 201.

7 F. Calogero, J. Math. Phys. 10 (1969) 2191, 2197; J. Math. Phys. 12 (1971) 419.

8 J.M. Leinaas and J. Mirheim, Phys. Rev. B 37 (1988) 9286; A.P. Polychronacos, Nucl. Phys. B 324 (1989) 597; F.D.M. Haldane, Phys. Rev. Lett. 67 (1991) 937.

9 J.A. Minahan and A.P. Polychronacos, Phys. Lett. B 326 (1994) 288.

10 V.V. Dodonov, V.I. Man'ko and L. Rosa, Phys. Rev. A 57 (1998) 2851.

11 M. Combescure, Ann. Phys. (N.Y.) 204 (1990) 113.

12 F. Cannata, G. Junker and J. Trost, in J. Riembelinski ed., Particles, Fields, and Gravitation, AIP Conf. Proc. 453 (Am. Inst. Phys., Woodbury 1998) 209; S. Seshadri, V. Balakrishnan and S. Lakshmibala, J. Math. Phys. 39 (1998) 838; D.J. Fernández, V. Hussin and L.M. Nieto, J. Phys. A 27 (1994) 3547; D.J. Fernández, L.M. Nieto and O. Rosas-Ortiz, J. Phys. A 28 (1995) 2693.

13 J.L. Cauley, Chaos, Dynamics, and Fractals: An Algorithmic Approach to Deterministic Chaos (Cambridge Nonlinear Science Series Vol. 2, Cambridge 1994).

14 P. Camiz, A. Gerardi, C. Marchioro, E. Presutti and E. Scacciatelli, J. Math. Phys. 12 (1971) 2040.

15 See for example, G. Junker and P. Roy, Phys. Lett. A 257 (1999) 113; G.S. Agarwal and S. Chaturvedi, J. Phys. A 28 (1995) 5747; D.M. Gitman and A.L. Shelepin, J. Phys. A 26 (1993) 7003.

16 A.O. Barut and L. Girardello, Commun. Math. Phys. 21 (1971) 41.

17 R. Koekoek and R.F. Swarttouw, The Askey-scheme of hypergeometric orthogonal polynomials and its $q$-analogue, Report 94-05, (Technical University Delft, 1994).

18 B.E. Palladino and P. Leal Ferreira, Rev. Mex. Fís. 44 (1998) 73. 